\title{
A Gesture Recognition Design Toolkit Everyday Gesture Library
}

\author{
Muhammad Haroon \\ Preston University, Islamabad
}

\author{
Malik Sikandar Hayat Khiyal \\ Faculty of Computer Sciences \\ Preston University, Islamabad
}

\begin{abstract}
Creating gestural recognition system is a challenging task which requires skills and updated applications. It is required for designer to be skillful and innovative in order to create interesting and acceptable gestures. Gesture Recognition Design Toolkit (GRDT) is set of tools designed to simplify the gesture creation process for non experts. From designing a new gesture, to suggesting the best fit, to test it for false positive activation, till final selection, are the step-by-step functions of Gesture Recognition Design Toolkit. Creating gestural recognition system, interface designers encounter many challenges throughout the process. Selecting the best gesture for a particular motion with low probability of false positives is one of many. This study explores ways to improve the functionality and enhance the efficiency of gestural library using machine learning techniques and data mining
\end{abstract}

\section{General Terms}

Multiple Action Gesture Interface Creation, Human computer interaction, Symbolic aggregate approximation, Nearest neighbor dynamic time wrapping, Hidden markov model, Dynamic time wrapping, Extract, transform and load

\section{Keywords}

Gesture design toolkit (GRDT), Motion gesture recognition, Every day gesture library, Recommender system, False positives.

\section{INTRODUCTION}

To communicate among each other people first establish a connection to make sure that other person is now ready to receive message and acknowledge them [1]. Same is the case when humans try to communicate with machines. This study suggests that a proper connection should be established before any gestural activity with the device. This process surely reduces the probability of occurrence of false positive during normal body motion. Problem that can come up is the acceptance by the user because establishing a connection and then entering a gesture takes more time as compared to normal input mechanism. This difficulty is faced by most designers who are not domain experts.

Gestures are short and easily recognized by a computer but their creation is tough. On the first hand to create a particular gesture that is distinguishable from user's normal movement is a challenging task [2]. On the other hand interface designers are not domain expert in gesture recognition which needs more skills than interface designing. Gesture designing can be done using of the shelf design tools easily available in the market but they are not full fledge design tools [3]. For instance some tools offer gesture designing and some are capable of testing a gesture for occurrence of false positive and they are slow in creating and testing. Testing which involves repeated process of load and check can be disappointing if the system works slow and takes a lot of time. Gesture Recognition Design Tool works with EGL- Everyday Gesture Library and machine learning techniques to speed up the process of load and check.

Study includes that EGL- Everyday Gesture Library is an important tool in motion gesture designing which is used to store gestural data. Focus of this study is "suggesting and testing of motion gestures to avoid false positives during the use". This suggesting and testing must be fast so it saves time. Speed can be improved by deploying machine learning techniques like Hidden Markov Model and K-Nearest model.

\section{PROBLEM STATEMENT}

To test a hand motion related gestures using GRDT (Gesture Recognition Design Tool) a gesture is designed, tested and selected for devices capable of gestural interaction?

\section{LITERATURE REVIEW}

This section reviews some of the papers from the scope of the study like creation of gesture, testing of gesture, saving time while testing different gestures.

3.1 Design tool for camera based interaction talks about camera based interactions [4]. The image processing with crayons is a tool for creating new camera based interfaces using a simple painting metaphor. Traditional machine learning algorithm has been modified to accommodate the rapid response time required for an interactive design tool, as shown in figure 1

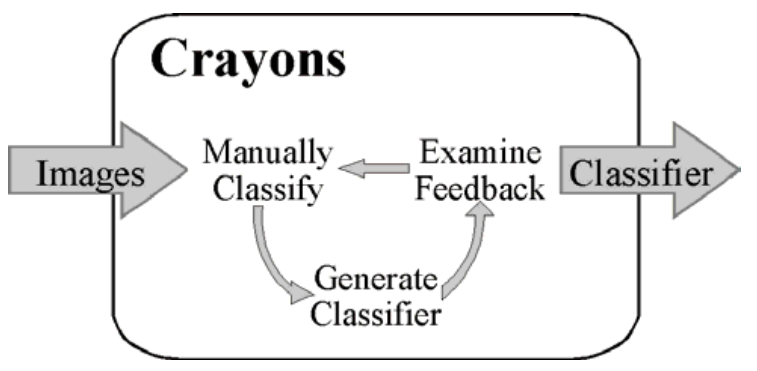

Fig 1: Crayons illustrate how interactive matrix language can be used to free designers from the knowledge of machine learning and image processing [4]. 


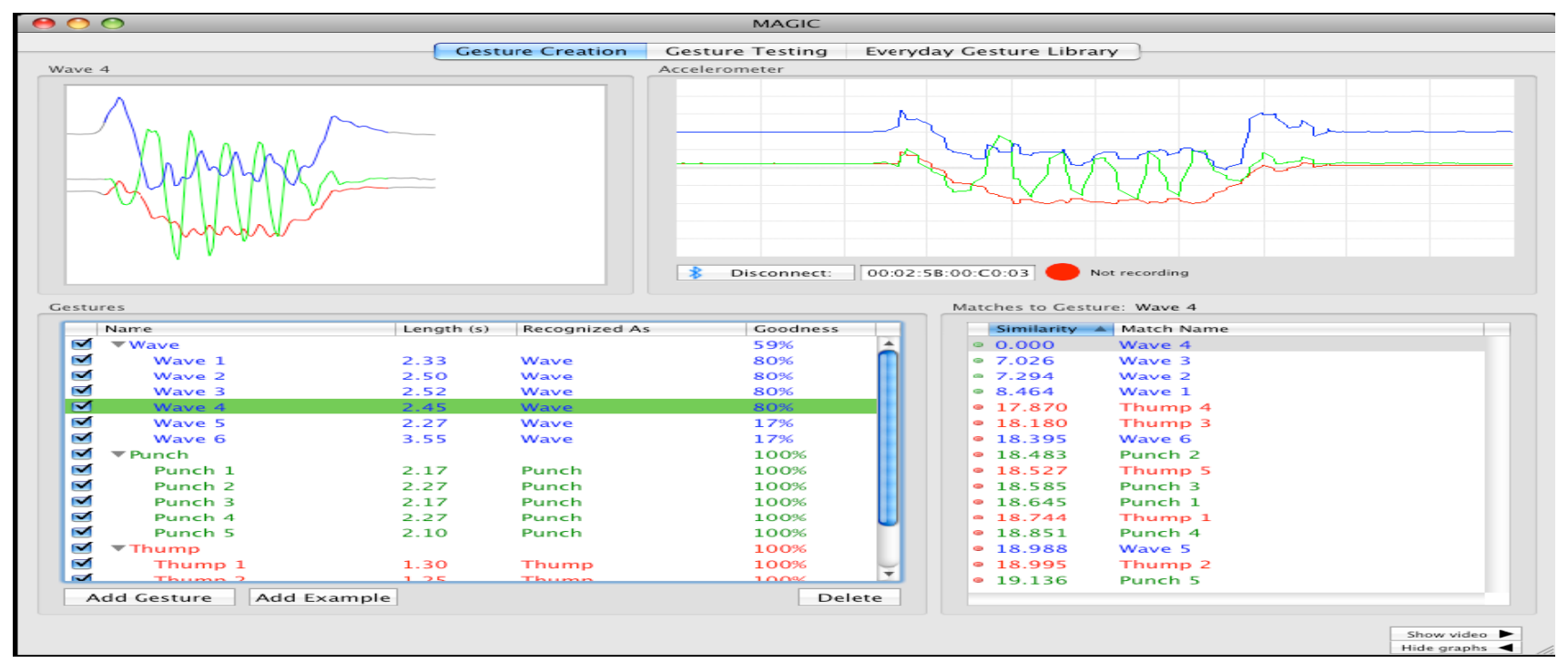

Fig 2: Gesture creating tab. A: recorded gesture; B: live sensor view; C: gesture example; D: sorted list [3].

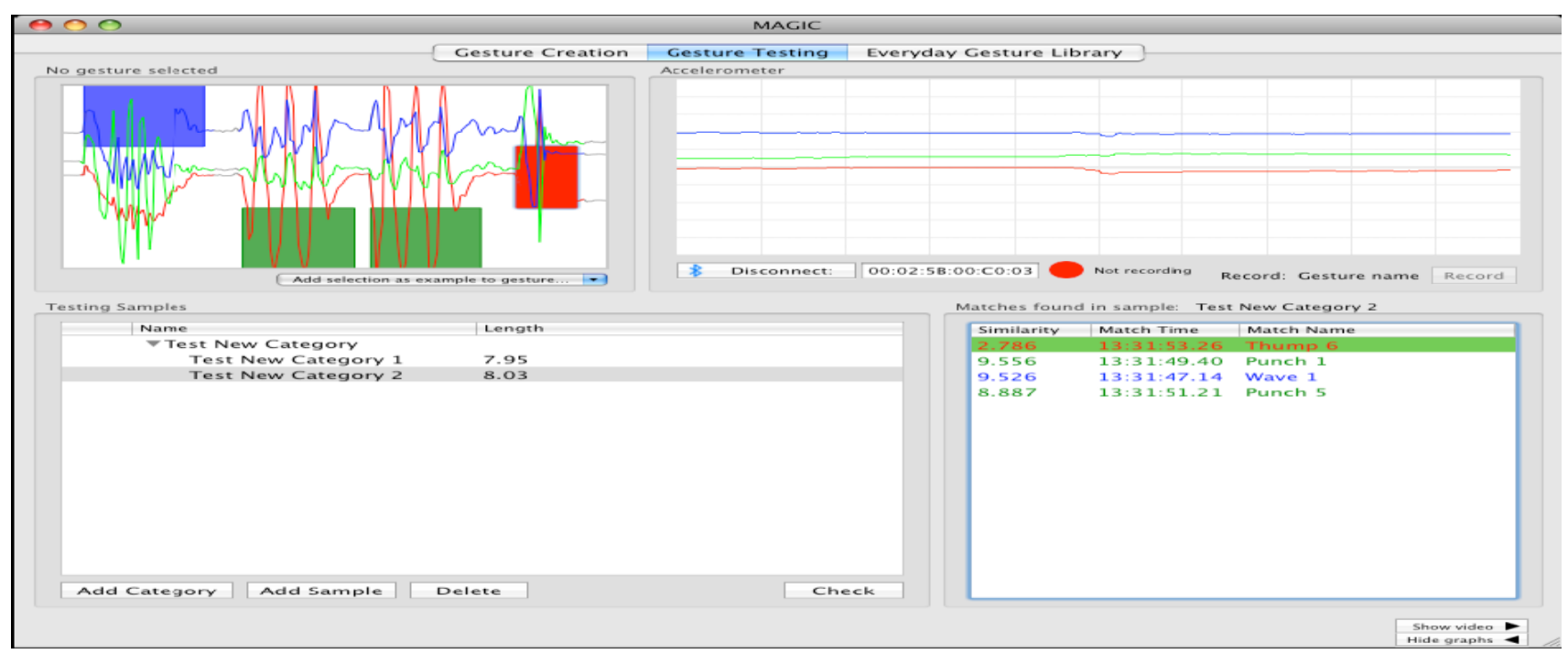

Fig 3. The Gesture Testing tab. A: recorded test sample view with boxes highlighting matches; B: live sensor view; C: list of test samples; D: list of gestures matching currently selected test sample [3].

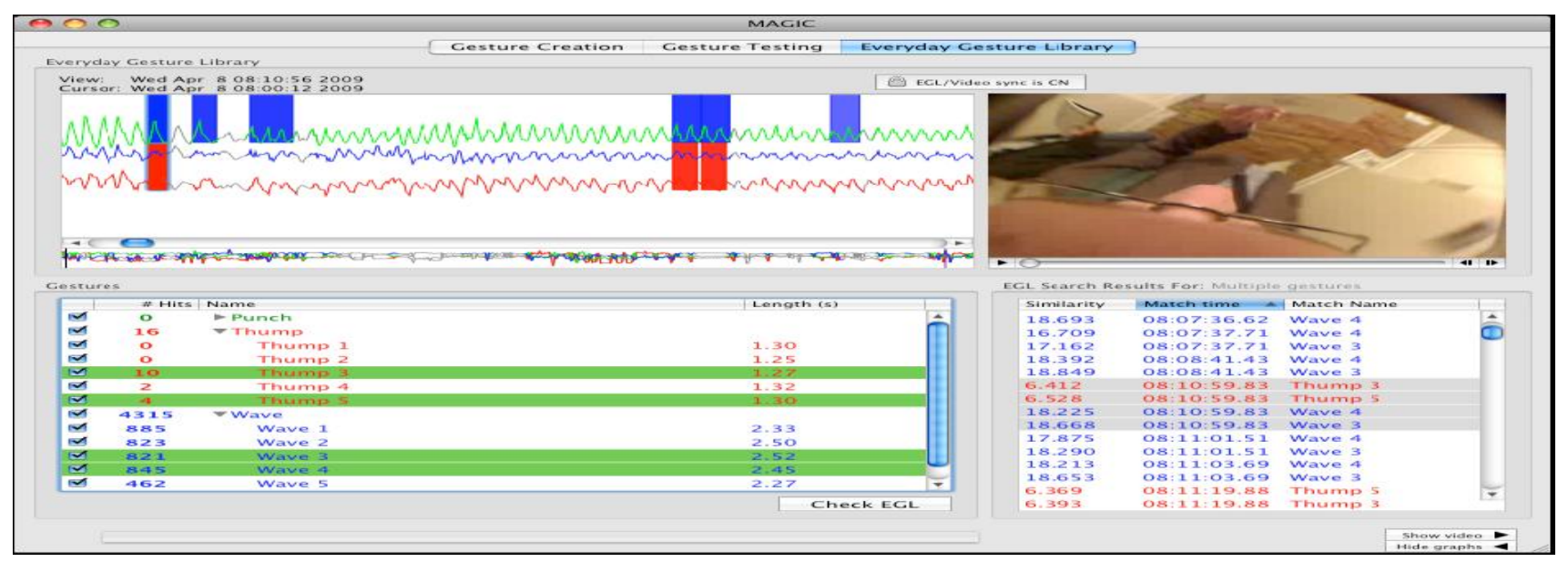

Fig 4. The Everyday Gesture Library tab. A: EGL view with boxes highlighting gesture occurrences; B: EGL video synchronized to EGL view; C: gestures with number of occurrences in EGL (\# Hits); D: list of occurrences in EGL for selected gestures [3]. 
3.2 Direct manipulation interface talks about advantages and disadvantages of direct manipulation interface [5] Phenomenon that concerns the relation between the input and output vocabularies of the interface language. The analysis of execution and evaluation process explains why there is difficulty in using a system. Problems with direct manipulation are the matching of levels of thought to the interface language and semantic directness [5].

\subsection{MAGIC (Multiple Action Gesture Interface} Creation): presents a tool that helps interface designers in pattern recognition. This paper also discusses how to effectively test gestures to ensure that they are not unintentionally activated by user's normal movements [3]. Creating a gesture system is a staged process that has three basic stages.

3.3.1 Gesture Creation: At first stage designer understands the requirement and decides what functionality to be controlled by the gesture. Designer designs the gesture classes and gesture examples. A gesture class represents one kind of movement, such as "shuffle a song". Designers normally create several examples for each class in order to account for variation in how a user might make a gesture, as shown in figure 2

3.3.2 In the second stage (Gesture Testing), the designer determines how motions by the user will trigger the functionality on the device. Designers ensure that only intended movements activate functionality. Then confirms that the gestures work reliably, and performs initial user testing. The gesture testing tab is shown in figure 3 .

One potential problem when creating gestures intended for everyday use is that the end user may perform same motion for different actions, which possibly lead to an unwanted activation of functionality. For example, in I pod music machine shaking your hand will trigger the device to shuffle the song. If user unintentionally shakes his hand, it will shuffle the song, which is false positive right there that's why testing gesture is very important. This testing can be done using EGL library, as shown in the figure 4.

The everyday Gesture library interface is similar to gesture testing tab, however it represents the set of users, allowing designer to determine if the created gesture is same as the everyday movement.

3.3.3 At Final Stage, the designer performs final user testing and deploys the finished product [3].

Problem of magic are as follows:

i. Magic primarily designed as an HCI tool, the system used nearest neighbor method with dynamic time wrapping distance measure.

ii. Relative false positive rates predicted in user studies were not compared to the actual false positive rates of a gesture recognizer running in the field.

iii. Long time needed to search for potential hint in a data base of EGL. If found to trigger during testing, the gesture set has to be changed.
3.4 Preface to the special issue on user interfaces for recommender systems: recommender system is a useful searching system as it uses the preferences and needs of the user. According to the paper recommender system has three basic approaches

\subsubsection{Collaborative technique}

\subsubsection{Content- based recommendation}

\subsubsection{Knowledge-based recommendation}

In collaborative technique system recommends the search result by checking the nearest neighbor according to its behavior. In Every day gesture library it can help designer to suggest gestures that are close to behavior given by the designer. Content-based recommendation system searches the required item by the help of preferences provided by the user and its similarities with item's description. Knowledge-based recommendation systems, finds the items by using 'user defined requirements [6]

3.5 Magic Summoning: Towards automatic suggesting and testing of Gestures with low probability of false positive during use: presents Magic Summoning, a web based tool that addresses the problem of distinguishing user's normal movement and selected gesture. Using large data base of sensor data and quantized via SAX) to enable quick searching [2].

Magic summoning tool kit has two software components First component is gesture recorder running on an android device. Second component is 'magic' web based application [2].

The recorder automatically estimates when gesture starts and when it ends. After example is recorded, recorder uploads the example to the web. Then designer evaluates the gesture in web application using nearest neighbor dynamic time wrapping (NN-DTW), as shown in the figure 5.

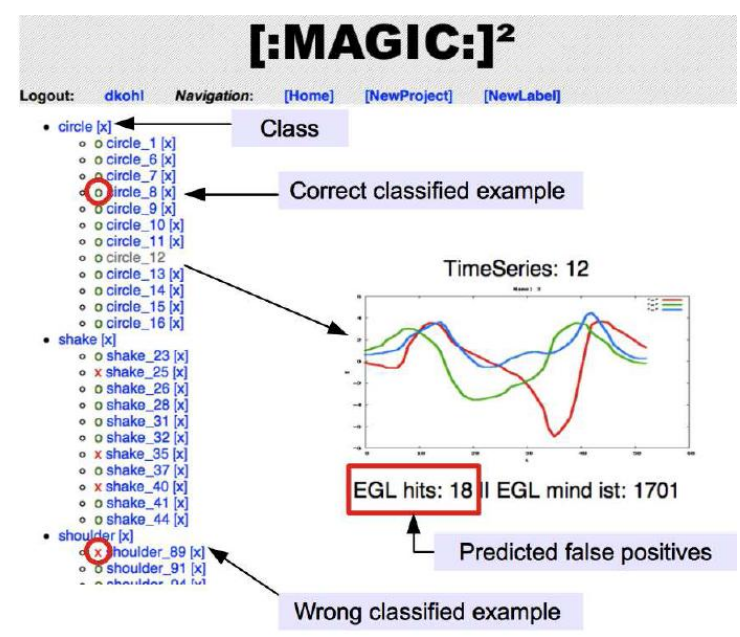

Fig 5: Magic summoning [2].

Movements used for recognition are pre-recorded from environment using sensor recorders and accelerometer, allowing the designer to determine if the created gestures is different in action with end-users' everyday movements. This pre-recorded data stored in library is called the "Everyday Gesture Library" (EGL). Everyday Gesture Library is an important tool in motion gesture designing. EGL is a space where we gather a large database of gestural data. Data 
captured from environment about the user's every day motions. Symbolic Aggregate approximation (SAX) enables EGL to search quickly from a huge set of data base [2]. SAX suggests gestures with a low likelihood of false triggering. Suggested gestures are ordered according to simplicity, freeing the interface designer to focus on the user experience, as shown in figure 6 .

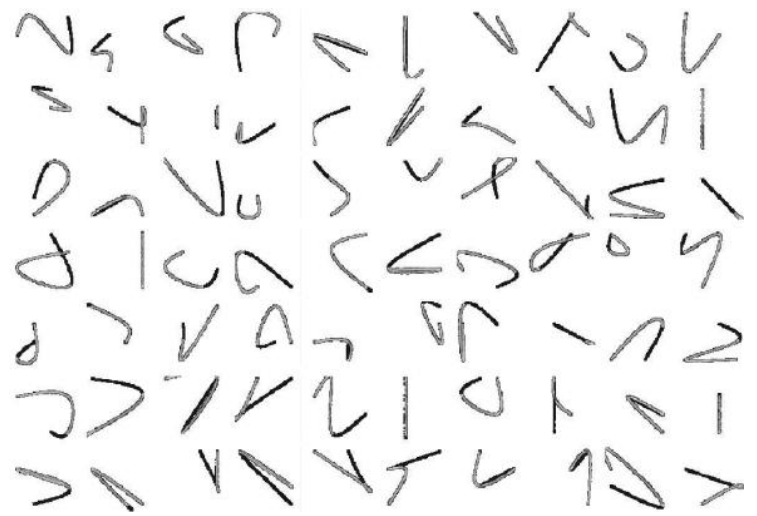

Fig 6: gesture gallery [2].

3.6 The Gesture Recognition Toolkit_is a cross-platform open source $\mathrm{C}++$ machine learning library specially designed to help non specialists in gesture recognition.

Large number of machine learning application are available that are designed for offline analysis of the data. And some machine-learning libraries that can be used to prototype real time systems are also accessible. In either case users need to build their system in $\mathrm{C}++$ to compute and execute the data

Gesture recognition toolkit addresses this issue with the ease of accessibility, flexibility, choice, customizability and real time support [7]. The example of user code is as follows:

\section{CODE EXAMPLE}

//Setup a custom recognition pipeline

1: GestureRecognitionPipeline pipeline;

2: pipeline.addPreProcessingModule( MovingAverageFilter( 5 ));

3: pipeline.addFeatureExtractionModule( FFT( 512 ));

4: $\quad$ pipeline.addFeatureExtractionModule(

MyCustomFeatureAlgorithm());

5: pipeline.setClassifier( Adaboost( DecisionStump()) );

6: $\quad$ pipeline.addPostProcessingModule(

ClassLabelTimeoutFilter( 1000 ));

//Load a labeled data set from a CSV file and train a classification model

7: ClassificationData trainingData;

8: trainingData.load( "TrainingData.csv" );

9: bool success $=$ pipeline .train $($ trainingData $)$;

I/The following lines would be called each time the user gets a new sample

10: vector< double > sample = getDataFromSenor () ; //Custom user function

11: pipeline.predict( sample);

12: unsigned int predictedClassLabel = pipeline.getPredictedClassLabel();

13: double maxLikelihood =

pipeline.getMaximumLikelihood(),

[7]

The advantages of GRT are as follows:

- Provides minimal code for user thus reducing the need for difficult coding.
- Uses an object oriented modular architecture like feature extraction, clustering, classification, and regression.

- Features broad range of machine learning algorithms such as Decision trees, DTW, HMM, K-nearest model, Naïve bayes, and multilayer perceptrons.

- Supports offline analysis on pre-recorded data sets and is also designed to help user move from offline analysis phase to real time recognition phase.

\section{METHODOLOGY}

This research is Qualitative, discussing and suggesting a tool to support different stages of gesture designing. It also helps non experts in pattern recognition to design and test a gesture. Gesture recognition design tool will help non expert to build their own gestural system from the scratch to a fully functional gesture. In literature review this study includes ideas and findings of different researches. On the basis of literature review, GRDT is proposed which is the combination of techniques to form an enhanced platform for designing, testing, and launching a gesture.

\section{DESIGN STRATEGIES}

Before designing a gesture designer must do consider the following

- Gestures should be easy to remember

- Be socially acceptable with high average of quality

- Must not trigger by users normal movement which is also called false positive activation.

\subsection{False Positives}

False positives or Mistakes are common problem in motion gesture designing. In development phase gesture seems fine but triggers false activation during testing or deployment. Designer has to restart the development making it more costly. For example, in I pod music machine shaking your hand will trigger the device to shuffle the song [2]. If user unintentionally shakes his hand, it will shuffle the song, which is false positive right there.

MAGIC is a tool for false positive prediction and prevention that can be used during the interface design process. With the help of every day Gesture Library match the motion gesture to predict the gesture's suitability [2]

\section{RESULTS}

GRDT is an open source platform to design a gesture, test its suitability and false activation and finally launch a gesture. Once completed, GRDT will be available online to perform above mentioned stages of interaction designing system. Also will include most of the application needed throughout the process.

To design a still gesture, pen based creation and camera based image processing techniques can be used by the user at its end. Or online gesture creation tools are included in the GRDT like quail etc.

To design a motion gesture GRDT uses the Everyday gestures library that keeps the record of motion gestures recorded from the environment using android devices, accelerometer and motion sensing devices. Storing the data is done at the server's end rest is done at the user's end.

Storing a particular data in a huge storage space to extract when needed is technical. In GRDT data is stored under appropriate name, folder using ETL (Extract, transform and load) technique of Data Ware housing. 
In order to find a required example of a gesture Data Mining is very useful method. Once search is done and whole lot of gesture class related to a particular motion is found next step is selection. To select GRDT has couple of options to choose from like classification method and $\mathrm{K}$ - nearest method. Another option in which user records the gesture in a real time using automated recorder that automatically estimates when gesture starts and ends to upload it to a web based application that checks the suitability of that gesture. Final phase of deploying a gesture will be done by the user after downloading it from the GRDT, as shown in fig 7.

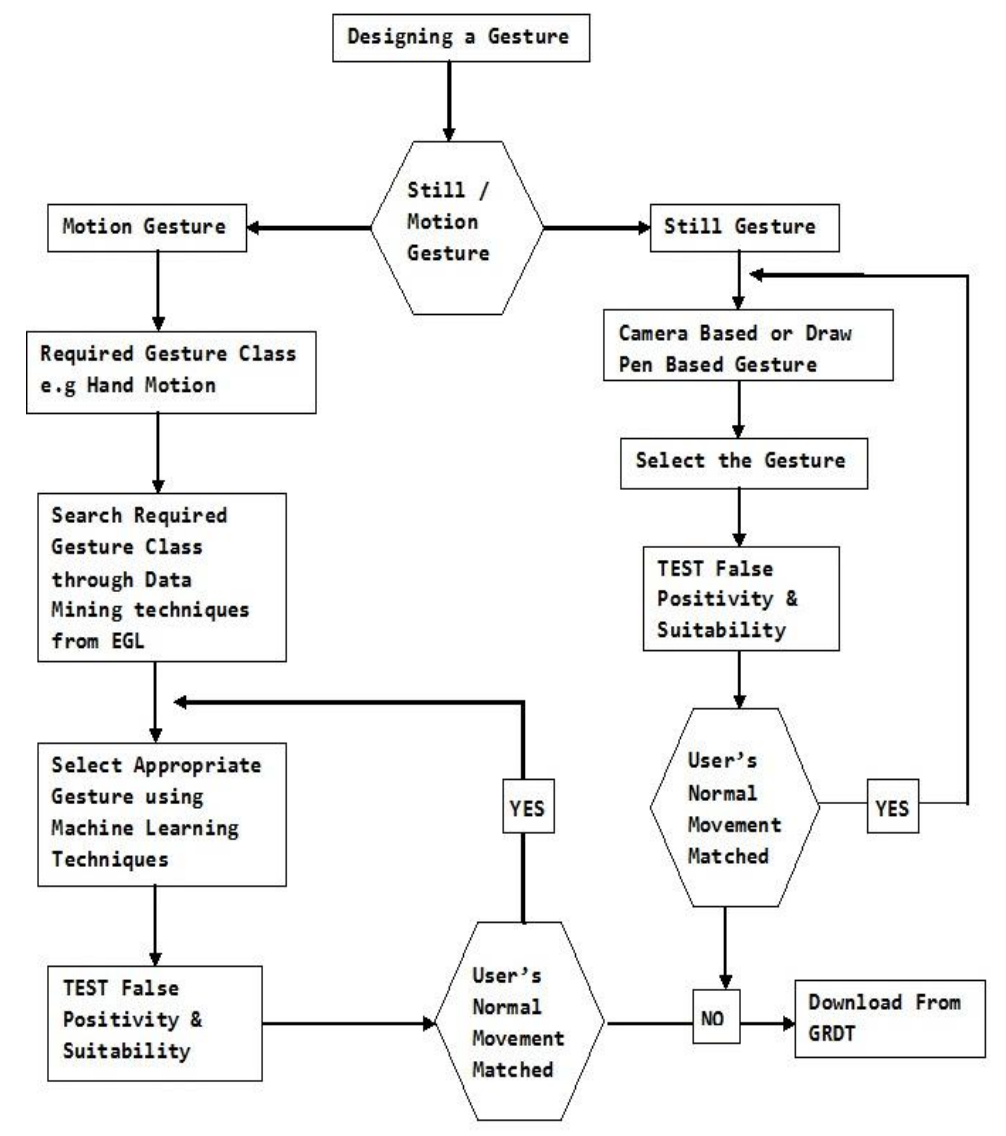

Fig 7: framework of GRDT

\section{CONCLUSION}

At this point of time GRDT is in a planning phase after a wide review of related literature. Future scope includes a web site of Gesture recognition design toolkit. GRDT is a platform that holds all the applications and helping materials in order to help a non expert in creating a gesture.

\section{REFERENCES}

[1] Rubine, D.H: The Automatic Recognition of Gestures. Ph.D. Thesis. Carnegie Melon University, CMU-CS-91-202 (1991).

[2] Kohlsdorf, D., and Starner, T: MAGIC Summoning: Towards Automatic Suggesting and Testing of Gestures with Low Probability of False Positives during Use. Journal of Machine Learning Research 14: 209-242 (2013).

[3] Ashbrook, D., and T Starner: MAGIC: a motion gesture design tool. In Proceedings of the ACM SIGCHI Conference on Human Factors in Computing Systems, pages 2159-2168, 2010.
[4] Fails, J.A., and Olsen, D,R: A Design tool for camera- based interaction. In proceeding of the ACM conference on human factor in computing systems (CHI), pages 449-456, 2003

[5] Edwin, H., James, H., and Donald, N.: 1985 Direct manipulation interfaces. Human-Computer Interaction, volume 1, pp.311-338.

[6] Felferning,A., Burke, R., and P Pu: Preface to the special issue on user interfaces for recommender systems. Journal of User Modeling and User-Adapted Interaction 22: 313316 (2012).

[7] Gillian, N., and Paradiso, J. A: The gesture recognition toolkit. Journal of Machine Learning Research 15: 34833487 (2014).

[8] Kohlsdorf, D. Starner, T., and Ashbrook, D., 2011 MAGIC 2.0: A web tool for false positive prediction and prevention for gesture recognition systems. In Proceedings of the International Conference on Automatic Face and Gesture Recognition, pages 1-6, Washington, District of Columbia. 